\title{
Das «biochemische» Heilverfahren nach Wilhelm Heinrich Schüßler und seine Weiter- entwicklung durch Dietrich Schöpwinkel
}

Axel Helmstädter

\section{Summary}

The German Physician Wilhelm Schüßler (1821-1898) developed a healing system called "Biochemie". Basically it had merely the character of a substitution therapy with mineral compounds and was based on physiological considerations by Jakob Moleschott (1822-1893) and Justus von Liebig (1803-1873) which were fundamental for the development of the scientific discipline of biochemistry as well. Schüßler himself, however, who had been educated in homoeopathy, introduced more and more immaterial or "biodynamic" explanations for the therapeutic effect of the mineral salt preparations. This attempt was continued by his successors, namely by Dietrich (called Dieter) Schöpwinkel (1876-1946) who enriched the system with new preparations as well as several theoretical aspects strongly related to the dynamic approach.

Keywords: Biochemie; Wilhelm Heinrich Schüßler; Jakob Moleschott; Dieter Schöpwinkel; alternative and complementary medicine

\section{Zusammenfassung}

In der zweiten Hälfte des 19. Jahrhunderts entwickelte der deutsche Arzt Wilhelm Schüßler (1821-1898) seine «biochemische Heilmethode», die ihren Ursprung in physiologisch-chemischen Vorstellungen von Jakob Moleschott (1822-1893) und Justus von Liebig (1803-1873) hatte und einer Substitutions-

Priv.-Doz. Dr. Axel Helmstädter, Institut für Geschichte der Pharmazie, Universität Marburg, Roter Graben 10, D-35032 Marburg (axel.helmstaedter@staff.uni-marburg.de). 
therapie mit anorganischen Salzen nahekam. Bereits der als Homöopath ausgebildete Schüßler selbst postulierte jedoch darüber hinausgehende immaterielle Wirkmechanismen, ein Ansatz, der von seinen Anhängern ausgebaut wurde. In dieser Hinsicht ragt der Heilpraktiker Dietrich, genannt Dieter, Schöpwinkel (1876-1946) hervor, der die «biochemische Heilweise» in den 1920er und 1930er Jahren durch neue Präparate sowie theoretische Ansätze stark erweiterte. Heute werden Schüßler-Salze vor allem unter dem Aspekt beworben, die Lebenskraft des Körpers stärken zu können; damit reiht sich die Methode in die «biodynamistischen» Verfahren der Komplementärmedizin ein.

Das «biochemische Heilweise» genannte, komplementärmedizinische Verfahren des Oldenburger Arztes Wilhelm Heinrich Schüßler (1821-1898) erfreut sich gegenwärtig einer grossen Beliebtheit bei Patienten ${ }^{1}$. Seine heutige Ausprägung erhielt das Verfahren nicht nur durch den Gründer selbst, sondern auch durch Nachfolger und Protagonisten im Verlauf des 20. Jahrhunderts, von denen Dietrich (genannt Dieter) Schöpwinkel (18761946) der prominenteste ist. Sein Beitrag zur Ausformung der «biochemischen Heilmethode» wird an dieser Stelle erstmals beschrieben, nachdem ein Überblick über die Geschichte des Schüßler-Verfahrens gegeben wurde.

\section{«Biochemie» nach Schüßler}

Die sogenannte «Biochemie» des Oldenburger Arztes Heinrich Schüßler (1821-1898), die aus heutiger Sicht nicht mit der naturwissenschaftlichen Disziplin gleichen Namens verwechselt werden darf, wurde bereits vielfach beschrieben ${ }^{2}$. Dies gilt auch für den Lebensweg des Initiators, der aus (Bad) Zwischenahn bei Oldenburg stammte. Schüßler agierte als Schreiber und Nachhilfelehrer, als er auf die Homöopathie aufmerksam wurde und sich entschloss, ein Medizinstudium aufzunehmen, obwohl er kein Abitur nachweisen konnte. 1852 gelang es ihm mit finanzieller Unterstützung aus der

1 Ein Indiz dafür ist, neben der Erfahrung in Apotheken, die Tatsache, dass gängige Buchhandelskataloge mehr als 50 erst in den letzten Jahren erschienene Titel zur Therapie mit Schüßler-Salzen aufweisen.

2 Zu Leben und Werk vgl. beispielsweise Platz 1921; Lampert 1953, 35-46; Winter 1971; Jütte 1996, 221-228; Ulpts 1998 und Borchardt 2001. Schüßler und sein Heilsystem wurden in der naturwissenschaftlichen wie komplementärmedizinischen Literatur viel beachtet und kontrovers diskutiert. Eine Bibliographie ohne Vollständigkeitsanspruch nennt ungefähr 1000 Bücher, Zeitschriftenartikel und Buchbeiträge: Gefken 1998. 
Familie unter ungeklärten Umständen ${ }^{3}$, in Paris immatrikuliert zu werden, ein Jahr später wechselte er - eventuell unter Vorlage eines gefälschten Reifezeugnisses ${ }^{4}$ - an die Universität Berlin. Eine praktische Tätigkeit als Homöopath in Oldenburg vor Beendigung des Studiums brachte ihm eine, später allerdings in einen Verweis umgewandelte Geldstrafe ein ${ }^{5}$. Die Universität Gießen promovierte Schüßler noch vor Abschluss des Studiums ebenfalls unter fragwürdigen Umständen. Weil er angab, seine Einberufung als Militärarzt stehe unmittelbar bevor, erliess man ihm die Abfassung einer Dissertation und gab sich mit einem Kolloquium und der Zahlung von Bearbeitungsgebühren zufrieden ${ }^{6}$. Die Zulassung zur ärztlichen Staatsprüfung in Oldenburg wurde ihm hingegen unter Hinweis auf das fehlende Abitur, die fehlende Dissertation und mangelnde Studiennachweise verwehrt ${ }^{7}$. Daraufhin komplettierte er sein Medizinstudium durch drei zusätzliche Semester in Prag und holte 1857 in Oldenburg das Abitur nach. Im gleichen Jahr bestand er dann das medizinische Staatsexamen; die ärztliche Approbation erhielt er am 2. Januar 1859 und liess sich daraufhin als homöopathischer Arzt in Oldenburg nieder ${ }^{8}$. In den 1870er Jahren formulierte Schüßler die Prinzipien seines Heilsystems, zunächst in einem Aufsatz in der <Allgemeinen Homöopathischen Zeitung ${ }^{9}$, hauptsächlich aber in dem zunächst nur 16 Seiten umfassenden Werk <Eine abgekürzte Therapie, gegründet auf Histologie und Cellular-Pathologie ${ }^{10}$. Winter führt die Begründung eines eigenen Heilsystems auf eine gewisse Stagnation in der Homöopathie zurück, die mit zunehmenden Erkenntnissen der naturwissenschaftlichen Medizin einherging. Wie schon im Titel zu Schüßlers Hauptwerk anklingt, bezog der Oldenburger Arzt vor allem die Zellularpathologie seines Zeitgenossen Rudolf Virchow (1821-1902) in seine Überlegungen ein. Tatsächlich machte sich der eingefleischte Homöopath durch diese - und so wurde es aufgefasst - Abkehr von der reinen homöopathischen Lehre unter deren Anhängern viele Feinde. In einem in der Fachpresse leidenschaftlich geführten Dialog betonte er letztlich die Eigenständigkeit seiner «biochemischen» Lehre, die kein homöopathisches System sei, sondern sich auf «physiologisch-

3 Winter 1971, 25.

4 So mutmasst Winter 1971, 26.

5 Winter 1971, 27.

6 Winter 1971, 28f.

7 Nach Winter 1971, 29f., schlugen in der Folge Versuche fehl, das Abitur im Ausland (Prag) oder unter erleichterten Bedingungen nachzuholen.

8 Winter 1971, 30-33.

9 Schüßler 1873.

10 Die <abgekürzte Therapie> erschien erstmals 1874 und erlebte nach Gefken 1998,13-26, bis 193357 Auflagen sowie zahlreiche Nachdrucke und fremdsprachige Ausgaben. Ab 1880 war ein Umfang von etwa 60-70 Seiten erreicht, der fortan konstant bleiben sollte. 
chemische Vorgänge» beziehe ${ }^{11}$. In späteren Auflagen der <abgekürzten Therapie> bezog er sich ausdrücklich auf damals neuere Erkenntnisse der Physiologie, vor allem auf die Schrift «Kreislauf des Lebens» des Niederländers Jakob Moleschott (1822-1893) ${ }^{12}$. Dieser hatte auf die Bedeutung anorganischer Substrate in der Physiologie, besonders auch auf zellulärer Ebene, hingewiesen. Die Erkenntnisse korrelierten mit den agrikulturchemischen Forschungen Justus von Liebigs (1803-1873) mit ihren praktischen Auswirkungen auf den Einsatz anorganischer Düngemittel und die Steigerung landwirtschaftlicher Erträge. Schüßler selbst stellte eine Analogie zur Landwirtschaft her:

Die Konstitution der Zelle ist durch die Zusammensetzung ihres unmittelbaren Nährbodens bedingt, wie das Gedeihen der Pflanze durch die Beschaffenheit des im Bereiche ihrer Wurzelfasern befindlichen Bodens. Der Agrikulturchemiker spricht vom $<$ Gesetz des Minimum >, nach welchem der im Boden im Minimum vertretene Pflanzennährstoff als Dung bezeichnet werden muß. ${ }^{13}$

Der Oldenburger Arzt hielt zudem die Beschränkung auf Anorganika für eine willkommene Vereinfachung der homöopathischen Therapie und beschränkte sich auf den Einsatz von zunächst zwölf, später nur noch elf anorganischen Salzen, die er «Funktionsmittel» nannte ${ }^{14}$. Sie sollten «durch Substitution des erlittenen Defizits durch einen gleichartigen Mineralstoff $\gg^{15}$ die korrekte Funktion einer erkrankten Zelle wiederherstellen. Richtlinien zur Auswahl der Mittel im Einzelfall bezog Schüßler teilweise aus homöopathischen Arzneimittelprüfungen, aber auch aus anderen Kriterien, wie beispielsweise dem Aussehen des Zungenbelages seiner Patienten ${ }^{16}$. Erst Nachfolger Schüßlers erweiterten die ursprüngliche Mittelpalette durch weitere Präparate, heute gibt es weitere zwölf «Ergänzungsmittel» ${ }^{17}$.

11 Vgl. hierzu Jütte 1996,222. In seinem Lehrbuch betonte er: «Mein Heilverfahren ist aber kein homöopathisches, denn es gründet sich nicht auf das Ähnlichkeitsprinzip, sondern auf die physiologisch-chemischen Vorgänge, welche im menschlichen Organismus sich vollziehen.» Schüßler 1911, 4.

12 So z.B. in Schüßler 1882,1f., 8; Schüßler 1911,1; vgl. hierzu Jütte 1996, 222f.; Winter 1971, 45f.; Borchardt 2001, 64.

13 Schüßler 1911, 8. Hervorhebung im Original. Vgl. auch Schüßler 1882, 1.

14 Schüßler selbst beschlichen Zweifel hinsichtlich der Zubereitung aus Calciumsulfat, die er 1887 wieder aus der Liste eliminierte. Seine Anhänger mochten indes nicht darauf verzichten, so dass das Salz heute sogar als «relevantes Mittel der Biochemie» eingestuft wird. Vgl. hierzu Jütte 1996, 223, und Gäbler 1988, 10. Auch moderne Veröffentlichungen aus dem engeren Kreis der Anhängerschaft nennen nur die elf bei Schüßler selbst unumstrittenen Mittel; so Harnisch 1998.

15 Winter 1971, 3.

16 Vgl. hierzu Borchardt 2001, 66f.

17 Siehe unten sowie Gäbler 1988 und Borchardt 2001, 67. 
Die Heilweise wurde von Homöopathie und Schulmedizin gleichermassen angefeindet, fand aber eine grosse, in Vereinen organisierte Anhängerschaft $^{18}$.

Tabelle 1: Biochemische Funktionsmittel nach Schüßler.

\begin{tabular}{lll}
\hline Calcium fluoratum & Kalium phosphoricum & Natrium phosphoricum \\
Calcium phosphoricum & Kalium sulfuricum & Natrium sulfuricum \\
Ferrum phosphoricum & Magnesium phosphoricum & Silicea \\
Kalium chloratum & Natrium chloratum (= muriaticum) & (Calcium sulfuricum) \\
\hline
\end{tabular}

Idee und Konzeption des Schüßlerschen Heilverfahrens nehmen deutlich Bezug auf Mitte des 19. Jahrhunderts gewonnene Erkenntnisse über Stoffwechselvorgänge, Ernährung, Verdauung von Menschen, Tieren und Pflanzen, die beispielsweise in der sogenannten Agrikulturchemie Justus von Liebigs ihren Ausdruck fanden. Derlei Untersuchungen bildeten einen wesentlichen Katalysator der «physiologischen Chemie», die sich - bis etwa 1900 noch immer so genannt - im Laufe des 19. Jahrhunderts als eigene wissenschaftliche Disziplin zur Erforschung von Stoffwechselvorgängen in Lebewesen entwickeln konnte ${ }^{19}$. Der Terminus «Biochemie», der spätestens seit 1858 verwendet wurde, war zunächst Synonym für den im Sprachgebrauch dominierenden Begriff «Physiologische Chemie» ${ }^{20}$. Auch Schüßler verwendete bis zu seinem Tode die Begriffe praktisch gleichsinnig, wenngleich im Laufe der Zeit der Terminus «Biochemie» häufiger wurde. Der Titel von Schüßlers zentralem Werk lautete ursprünglich «Eine abgekürzte Therapie gegründet auf Histologie und Cellular-Pathologie». Hier tauchen beide Begriffe noch nicht expressis verbis auf, allerdings heisst es zu Beginn: «Die Grundlage meiner Forschungen waren die Histologie, die darauf bezügliche Chemie, die anorganischen Bestandteile der Gewebe und die physiologischen Wirkungen oder Functionen dieser Bestandteile.» ${ }^{21}$ Im Untertitel der 1881 erschienenen 6. Auflage heisst es dann «Anleitung zur Behandlung der Krankheiten auf biochemischem Wege», noch im gleichen Jahr (8. Auflage) «Biochemische Behandlung der Krankheiten». Unter bezug auf ein Zitat aus Moleschott schreibt Schüßler im Vorwort:

18 Bezüglich der verzweigten Geschichte der biochemischen Vereine, die in den 1920er und 1930er Jahren etwa 200000 Mitglieder zählten, sei verwiesen auf Winter 1971, 52-86; Jütte 1996, 226-228, und Karrasch 1998, 43-59.

19 Schling-Brodersen 2005, 182f.

201858 erschien das Lehrbuch 〈Compendium der Biochemie〉 des Österreichers Vinzenz Kletzinsky (1826-1882), der «Biochemie» indes als Unterdisziplin der Biologie ansah. Felix Hoppe-Seyler schlug 1877 vor, die sich verselbständigende Disziplin der «physiologischen Chemie» «Biochemie» zu nennen. Vgl. Teich 1989, 241, und Vöckel 2003, 7 und 156.

21 Schüßler 1875,5 . 
Die obigen Worte haben mich zu einem Studium der physiologisch-chemischen Wirkungen der anorganischen Stoffe des menschlichen Organismus veranlasst. In Folge dieses Studiums, welches ich unter Zugrundelegung der, die Gewebe-Chemie betreffenden Thatsachen vor neun Jahren begann, ist eine biochemische Therapie entstanden, welche hiermit in achter Auflage erscheint. ${ }^{22}$

Im 1898 verfassten, noch in der 37. Auflage von 1911 enthaltenen Vorwort wird davon gesprochen, das Heilverfahren gründe sich auf «physiologischchemische Vorgänge, welche im menschlichen Organismus sich vollziehen» ${ }^{23}$. Während der Ausformung seines Heilsystems sprach Schüßler also ausdrücklich von dem jungen naturwissenschaftlichen Fach, das sich allerdings im Laufe des 20. Jahrhunderts sprunghaft zur bedeutsamen wissenschaftlichen Disziplin weiterentwickelte, mit der das - cum grano salis - unverändert gebliebene Heilsystem des Oldenburger Arztes heute praktisch nichts mehr gemein hat. Schüßlers «biochemisches» Heilverfahren und die naturwissenschaftliche Biochemie haben also gemeinsame Wurzeln; das Heilverfahren indes bedient sich nach wie vor physiologisch-chemischer Vorstellungen des 19. Jahrhunderts, wie sie beispielsweise von Moleschott und Liebig formuliert worden waren.

Der Bezug zu deren Ansichten als wesentliche Bausteine der frühen physiologischen Chemie rückt das Verfahren auf den ersten Blick in die Nähe zur Pflanzendüngung mit anorganischen Salzen im Sinne einer reinen Substitutionstherapie. In diesem Zusammenhang muss es schon als Inkonsequenz erscheinen, dass der Oldenburger Arzt - im Bannkreis der Homöopathie aufgewachsen und ausgebildet - eine Potenzierung seiner Mittel empfahl. Zwar blieb er dabei im Rahmen der Niederpotenzhomöopathie und beschränkte sich faktisch auf die Stärken D6 und (selten) D12; dennoch war er der Homöopathie so weit verhaftet, dass er sich von diesem Charakteristikum trotz substitutionstherapeutischer Ansätze nicht zu lösen vermochte. Indes setzte er sich erheblicher Kritik seiner homöopathischen Kollegen aus, denen er als Abweichler galt ${ }^{24}$. Die kleinen Dosen wurden unter anderem damit begründet, dass damit zwar Funktionsstörungen ausgeglichen werden könnten, die Funktionen gesunder Zellen aber nicht beeinträchtigt würden ${ }^{25}$. Die Salze sollten zudem nur aus gering konzentrierten Lösungen in die Zellen eindringen können, die ebenso wie die Interzellularflüssigkeit die Mineralien selbst nur in Konzentrationen enthielten, die der zweiten bis fünf-

22 Schüßler 1882, 4.

23 Schüßler 1911, 4. Es scheint, als sei der Oldenburger Arzt mit der Formulierung des Untertitels dem sicherlich wirkmächtigen Vorschlag Hoppe-Seylers aus dem Jahr 1877 gefolgt, was sich allerdings nicht belegen lässt.

24 Vgl. Jütte 1996, 224.

25 Deters 1926, 47. 
ten Dezimalpotenz entsprächen ${ }^{26}$. Reiztherapeutische Überlegungen spielen ebenfalls eine Rolle, so sollen Zellen auf pathogene Reize durch erhöhte Aktivität antworten und dadurch Mineralien verlieren, die therapeutisch substituiert werden müssen ${ }^{27}$. Die potenzierten Salze wiederum sollen zunächst einen Reiz auf die pathogen veränderte Zelle ausüben und deren «Molekularbewegungsstörungen» ausgleichen. Erst im zweiten Schritt werde dann das eigentliche Mineralstoffdefizit ausgeglichen ${ }^{28}$.

Der vordergründig mechanistische Ansatz wurde also bereits durch Schüßler selbst nicht durchgehalten, der sich - entgegen seinen Beteuerungen - weder vollständig von homöopathischen Traditionen zu lösen vermochte noch radikal auf die Seite der naturwissenschaftlichen Medizin schlug ${ }^{29}$.

\section{Dietrich (gen. Dieter) Schöpwinkel}

Nachfolger und Anhänger des Oldenburger Arztes entfernten sich immer weiter vom physiologisch-chemischen Aspekt der «biochemischen» Heilweise und postulierten verstärkt energetische Wirkmechanismen der eingesetzten Anorganika.

Hervorzuheben ist in diesem Zusammenhang der, wie er sich selbst nannte, «Forscher, Schriftsteller und praktische Biochemiker» Dietrich (gen. Dieter) Schöpwinkel (1876-1946), der Schüßlers Lehre in stark erweiterter Form als «solar-polar-biochemische Konstitutionstherapie» oder kurz «Polar-〈Biochemie»» propagierte und geradezu zur Weltanschauung erhob ${ }^{30}$. Schöpwinkel, zu dessen Lebensweg bislang nur spärliche Angaben zu ermitteln waren, wurde am 23.11.1876 in Hamm (Westfalen) geboren und kam 1909 nach Mülheim an der Ruhr. Dort wirkte er von 1902 bis 1909 und 1914 bis 1930, bevor er nach Düsseldorf verzog, wo er ein «Zentral-Laboratorium

26 Schüßler 1911,10. Es gab auch die Vorstellung, die Präparate seien um so wirksamer,je intensiver die Salze verrieben wurden, was natürlich für ihre homöopathische Potenzierung sprach. Vgl. Biochemische Central-Officin 1920, 2.

27 Winter 1971, 47.

28 Deters 1926, 48. Der Autor beruft sich - wie auch schon Schüßler 1911,11 - in diesem Zusammenhang auch auf die Arndt-Schulzsche Regel, wonach kleine Reize die Lebenstätigkeit anregen, mittlere sie fördern und starke sie eher hemmen: Deters 1926, 50f. Zur ArndtSchulzschen Regel vgl. Helmstädter 2004, 72-77.

$29 \mathrm{Zu}$ dieser Ambivalenz siehe Winter 1971, 39-45.

30 Bibliographien nennen gewöhnlich den Vornamen Dieter, auch er selbst nannte sich, wenn (wie meistens) nicht nur die Initiale «D.» angegeben ist, Dieter: Schöpwinkel 1937, 11. 
für Polar-Biochemie» betrieb ${ }^{31}$. Diesem angegliedert war ein Verlag, der unter anderem eine auf fünf Bände angelegte Schriftenreihe unter dem Titel «Die polar-biochemische Welterkenntnis» und die Zeitschrift «Der Mensch und sein Leben ${ }^{32}$ herausgab. Ab 1936 tragen seine Schriften den Ortsvermerk «Bad Honnef/Rhein», die Stadt, in die er 1935 verzogen war. Dort starb der «Polar-Biochemiker» am 2.3.1946 ${ }^{33}$.

Schöpwinkel erhob die «Biochemie» zum «Weltgesetz» und bezog sich ausdrücklich auf Schüßler, dessen Lehre er «biologisch erweitert» zu haben glaubte: Im Vorwort zum ersten Band der von ihm herausgegebenen Schriftenreihe heisst es:

Wir setzen voraus, dass der geneigte Leser sich mit der grundlegenden Schrift für Biochemie: 〈Eine abgekürzte Therapie〉 von Dr. med. Schüßler, vertraut gemacht hat. Ohne diese von unserm Meister geschaffene Grundlage halten wir jede praktische Betätigung auf biochemisch-therapeutischem Gebiete für sinnlos und leichtfertig. Nur die naturgesetzliche Keimkraft dieser genialen Grundideen bietet auch dem Jünger des Altmeisters die Gewähr, dass er sich ebenfalls zum Künstler und dadurch zum wahren Wegweiser und Helfer der leidenden Menschheit empor entwickelt. ${ }^{34}$

Die Erweiterung des Schüßler-Verfahrens bestand zunächst einmal darin, dass neben den Anorganika des Oldenburger Arztes noch eine Reihe meist physikalisch-therapeutischer Verfahren zugelassen, ja empfohlen wurde. Sie erwachsen nach Schöpwinkel erst auf Grundlage der «Polar-Biochemie», vergleichbar mit Ästen, die aus einem gemeinsamen Baumstamm spriessen:

Die aus der Wesenheit des universellen polar-biochemischen 〈Heil〉-Stammes hervorstrebenden Aeste, Zweige und Sprossen sind:Diät, Hygiéne, Körper- und Gesundheitspflege, die Phyto-Therapie (Pflanzenheilkunde), die physikalische Heilkunde (Licht, Luft, Wasser, Erde), die Aerotherapie (Atemheilgymnastik), Psychotherapie (Seelenheilkunde, Hypnotismus, Cueismus, Suggestion), Organo-Strahlentherapie (Magnetismus), Chromotherapie (Lichtfarbenbehandlung), Hämokinetik (Innere Atemdruck-Massage), Elektrotherapie (elektrische Schwachstrombehandlung), Homoeopathie. ${ }^{35}$

Dann führt der Heilpraktiker eine Reihe tatsächlicher oder vermeintlicher naturwissenschaftlicher Beobachtungen an, von denen Schüßler noch nichts

31 Stadtarchiv Mülheim an der Ruhr, Meldekartei; frdl. Mitt. v. 8.4.2004. Das Unternehmen bestand in Düsseldorf bis etwa 1941, frdl. Mittl. Stadtarchiv Düsseldorf vom 26.5.2004.

32 Das Periodikum erschien erstmals 1926 und bestand mit wechselnden Untertiteln bis mindestens 1936. Es war offizielles Organ der «Studiengesellschaft für philosophisch-biologischbiochemische Wissenschaften», des «Verbandes der geprüften praktischen Biochemiker» und der «Arbeitsgemeinschaft zum Studium und zur praktischen Auswertung der philosophisch-biologisch-biochemischen Wissenschaften», die wiederum Mitglieder von «Studiengesellschaft» und «Verband» umfasste. Im Untertitel des Jahrgangs 1936 nannte sich der Herausgeber Schöpwinkel nationalsozialistischen Ansprüchen genügend «Begründer der nordisch-gotisch-keltgermanischen polar-biochemischen Welterkenntnis und NaturHeilwissenschaft».

33 Frdl. Mitt. Stadtarchiv Bad Honnef v. 27.5.2004.

34 Schöpwinkel 1924, 7.

35 Der Mensch und sein Leben 1 1926, $23 \mathrm{f}$. 
wusste und die nun zur Erklärung der «biochemischen» Heilweise beitragen sollten. Auf diese Weise versteigt sich der «praktische Biochemiker» schliesslich zu einem kaum noch zu verstehenden Theoriegebäude, dessen Durchdringung noch durch zahlreiche Wortschöpfungen des Autors erschwert wird. Hier spielen insbesondere Erkenntnisse über den Ionencharakter in Lösung dissoziierter Salze und kolloidchemische Theorien eine Rolle. Diese werden verquickt mit Mutmassungen über die energetische Beeinflussbarkeit von Stoffwechselvorgängen, in denen der biodynamistische Charakter des als «wissenschaftliche Vervollkommnung und biologische Erweiterung der Dr. med. Schüßlerschen Biochemie $»^{36}$ gepriesenen Verfahrens besonders zum Ausdruck kommt. Die Verbindung zwischen physikalischer Chemie und immaterieller Lebenskraft ist angeblich bereits in der Bibel grundgelegt, denn erst der «lebendige (Sonnen-)Gottes-Odem» im göttlichen Schöpfungsakt habe auf der Basis anorganischer Materie das «biochemische Werden des Menschen ermöglicht $»^{37}$.

Schöpwinkel beruft sich ausdrücklich auf die Od-Lehre des Chemikers Karl von Reichenbach (1788-1869) ${ }^{38}$ sowie die Theorie der «N-Strahlen», die der französische Physiker René Prosper Blondlot (1849-1930) Anfang des 20. Jahrhunderts in die Diskussion brachte. Beiden Theorien ist gemeinsam, dass von Körpern eine imaginäre Strahlung ausgehen soll, deren Stärke mit den vitalen Funktionen des Körpers korreliert. Reichenbach entwickelte die Vorstellung einer das All und die gesamte Natur durchdringenden Universalkraft, die er zu den «Dynamiden» zählte und «Od» nannte. Nach Habacher erhielt er den Anstoss hierzu von einem Wiener Arzt, der bei einer Patientin im Dunkeln Lichterscheinungen als Reaktion auf eine magnetische Behandlung wahrgenommen haben wollte ${ }^{39}$. Reichenbach selbst war zu solchen Wahrnehmungen nicht in der Lage, glaubte aber «sensitiven» Personen, die vorgaben, in völliger Dunkelheit an Flammen erinnernde, Aura-ähnliche Lichtkränze um Lebewesen und Gegenstände zu erkennen. Den Namen, das «Lautzeichen für ein alles in der gesammten Natur mit unaufhaltsamer Kraft rasch durchdringendes und durchströmendes Dynamid», leitete Reichenbach von der germanischen Gottheit Odin ab, die er als Personifizierung «alldurchdringender Kraft» ansah.

Die nach der französischen Stadt Nancy benannten N-Strahlen sollten von lebenden, aber auch unbelebten, sonnenbestrahlten Körpern ausgehen

36 Schöpwinkel 1929a, 172.

37 Schöpwinkel 1924, 28.

$38 \mathrm{Zu}$ Reichenbachs Leben und zur Od-Lehre vgl. Helmstädter 2004, 183-187.

39 Habacher 1980, 108. 
und ebenfalls ein Mass für die Lebenskraft des Organismus sein ${ }^{40}$. Sie haben einen festen Platz in Schöpwinkels Therapiekonzept:

Die [...] polar-lebenschemischen Heilfaktoren sind mithin in erster Linie: das für jeden besonderen Fall spektralanalytisch modifizierte Sonnenlicht selbst [...], Luft und Wasser für jeden Fall besonders entsprechend polarisiert [...], die polarisierte Od- oder N-Strahlung des gesunden Menschen, der dem Menschen sympathischen Blüten und Pflanzen (Duftschwingungen) und der dem Menschen homogenen Metalle, Metalloide und Salze. ${ }^{41}$

Der «polar-biochemische Weg der Therapie» besteht in einer Rückführung der Lebenskraft ins Gleichgewicht durch möglichst «artgleiche Träger einer spektralen Sonnenenergie» ${ }^{42}$. Die «modern-kullinarische» (!) Zubereitung von Lebensmitteln führt nach Ansicht des Heilpraktikers zu einem Mangel an «anorganischen Energieträgern», was die «polar-biochemischen Wechselbeziehungen» im Körper stört. Dies suchte er dadurch zu beheben, dass man

die dem menschlichen Organismus homogenen vitalen, tellurischen und kosmischen Energieträger in ihrer ursprünglichsten Art und Form [...] in das organisierte System des animalischen Körpers einführt und dadurch auf den infolge einer pathologischen Störung gesetzmäßig umorganisierten Organismus restituierend und reorganisierend einwirkt. ${ }^{43}$

Dies geschah im polar-biochemischen Sinne durch Zufuhr von Elektrizität und magnetischer Energie als Verkörperung der N-Strahlung, Wasser, Luft und Erde sowie hauptsächlich durch die von Schüßler zur Therapie vorgeschlagenen Salze.

Indes erweiterte Schöpwinkel die Schüßlersche Mittelpalette ab 1913 durch weitere Präparate und trug damit wesentlich zur Ausdehnung des Therapiearsenals auf heute bis zu 24 Salze bei. In Schöpwinkels Werken ausführlich besprochen werden zunächst sechs, letztlich neun weitere Anorganika, bezeichnet als «neuerforschte, D. Schöpwinkelsche polar-biochemische Funktionsmittel» (Tab. 2). Der Autor verweist auf neue wissenschaftliche Erkenntnisse, die die Lebensnotwendigkeit weiterer Mineralien

40 Schöpwinkel 1929a, 87, 148, bezeichnet die N-Strahlen als «Lebens-Element ersten Ranges» und «N-Lebenskraft-Strahlen». Das von Blondlot beim Versuch, Röntgenstrahlen zu polarisieren, beobachtete Phänomen konnte alsbald als Täuschung entlarvt werden. Vgl. hierzu Ashmore 1993 und Zankl 2003,17-21. Dennoch dienen N-Strahlen bis heute als Erklärung okkulter Phänomene. Der Begriff wird nicht nur bei Schöpwinkel synonym zum «Od» gebraucht; vgl. Winckelmann 1956, 69. Das auch in der Diskussion um die Wirksamkeit homöopathischer Hochpotenzen kursierende Postulat der 〈Biophotonen〉 als Strahlungsemanation lebender Zellen wird noch immer mit der angeblichen Entdeckung Blondlots in Verbindung gebracht. Vgl. hierzu Bischof 1998.

41 Schöpwinkel 1929a, 140.

42 Schöpwinkel 1929a, 142.

43 Schöpwinkel 1929a, $146 f$. 
gezeigt hätten, und behauptet, sie «seit 1913 in die polar-biochemische Heilpraxis eingeführt» $\mathrm{zu}$ haben ${ }^{44}$.

Tabelle 2: Von Schöpwinkel zwischen 1913 und 1930 eingeführte Funktionsmittel.

\begin{tabular}{lll}
\hline Manganum sulfuricum & Kalium bromatum & Cuprum arsenicosum \\
Lithium chloratum & Kalium arsenicosum & Zincum chloratum \\
Kalium jodatum & Calcium sulfuratum & Kalium aluminium sulfuricum \\
\hline
\end{tabular}

Gewarnt wird vor Verwechslungen zwischen Calcium sulfuratum (Calciumsulfid) und Calcium sulfuricum (Calciumsulfat), dem von Schüßler selbst wieder verlassenen Mittel, das «vielfach irrtümlich empfohlen» worden sei ${ }^{45}$. Die drei Mittel Cuprum arsenicosum, Zincum chloratum und Kalium aluminium sulfuricum wurden wohl als letzte in die Therapie eingeführt; sie werden in der 1930 erschienenen 5. Auflage von Schöpwinkels zentralem Werk erst im Anhang ausführlich besprochen. Die 1929 erschienene «Verordnungs-Hinweis-Tabelle der polar-biochemischen Heil-Wissenschaft» führt indes schon alle 20 Mittel auf und wird durch eine eher verwirrende Fussnote ergänzt, die allerdings zeigt, dass sich der Heilpraktiker noch über weitere Mineralien Gedanken gemacht hatte:

Die von uns in verschiedenen Schriften als polarbiochemische Funktionsmittel erwähnten Mineralsalze Aurum natr. chlor., Argentum chlor., Barium chlor. und Strontium chlor. auch Strontium sulfat, sind aufgrund der durch unsere diesbzgl. langjährigen Forschungen gemachten Erfahrungen und Feststellungen für den harmonischen Aufbau des Organismus äußerst lebenswichtig. ${ }^{46}$

Die genannten Stoffe fanden jedoch letztlich keinen Eingang in die biochemische Therapie. Zu den heute als Ergänzungsmittel definierten zwölf Stoffen gehören die neun oben genannten Mittel Schöpwinkels und zusätzlich als Salze Nr. 22, 23 und 24 Calcium carbonicum, Natrium bicarbonicum und Arsenum jodatum. Ihr Einsatz geht wohl nicht auf Schöpwinkel zurück.

Der «Polar-Biochemiker» ergänzte seine auch guten Willens nur als verworren zu bezeichnende Theorie indes um eine Vielzahl weiterer, meist komplex zusammengesetzter Medikamente. So definierte er verschiedene

44 Schöpwinkel 1930, 39. Schöpwinkel war jedoch nicht der einzige Nachfolger Schüßlers, der unter Berufung auf neue Erkenntnisse bezüglich essentieller Mineralien und Spurenelemente die ursprüngliche Mittelpalette erweiterte. So bereicherte der Oldenburger Arzt Albert Reiff in den frühen 1920er Jahren die «biochemische» Heilweise mit den auch von Schöpwinkel propagierten Mitteln Manganum sulfuricum und Kalium jodatum sowie zusätzlich um Aluminium sulfuricum und Arsenum jodatum. Vgl. Reiff 1924, 11, und Reiff $1932,12$.

45 Schöpwinkel 1930, 47 f.

46 Schöpwinkel 1929b, 3. 
Konstitutionen des Patienten, für die es spezielle Mittel gab ${ }^{47}$. Er unterschied zwei «Grundkrankheitsarten», die «Anomal-Charakter-Typen A und B» mit erhöhter bzw. verringerter Aktivität. Diese wiederum brachte er in schwer nachvollziehbarer Weise in Verbindung mit atmosphärischen Gasen, woraus vier «Normal-Konstitutionen» mit entsprechenden Funktionsmitteln erwuchsen $^{48}$. So entstand beispielsweise die «Hydrogen-sanguinische Konstitutions-Anomalie» als

lebensgesetzwidrige Umorganisation der normalen 〈Frühlings-Konstitution〉 durch Verkümmerung der ursprünglich normalen, blauen, kühlenden, beruhigenden «N-LebenskraftStrahlung> infolge einseitig sanguinischer Bevorzugung der hier störend wirkenden, körperfremden, orange-gelb-grün strahlschwingenden (emanierenden) Wasserstoffverbindungen und Bildung von nur sanguinischen, optimistisch übertriebenen Gedankenketten. ${ }^{49}$

Mittel für diesen Veranlagungstyp war das «Hydrogenin», Patienten der drei anderen Konstitutionen wurden mit «Nitrogenin», «Oxygenin» oder «Carbogenin» behandelt. Diese Mittel waren Bestandteile der «elektrobiochemischen Serie» ${ }^{50}$; zur Auswahl wurden auch Geburts- und Zeugungsquartal des Patienten herangezogen. Eine zusätzlich definierte «Misch-Konstitutions-Anomalie» erforderte ein Arzneimittel namens «Pneumotebé», speziell für Diabetiker gab es das «polar-biochemische Diabetiker-Konstitutionsmittel Diabeticum». Alle diese Zubereitungen waren Gemische besonders aufbereiteter anorganischer Salze ${ }^{51}$.

Das polar-biochemische Laboratorium in Düsseldorf unter Leitung des Apothekers Emil von den Driesch ${ }^{52}$ stellte daneben noch zahlreiche weitere Produkte her, so 36 «Ergänzungspräparate» unterschiedlichster Art wie Badesalz, Gallensteinpillen, antiseptische Seife, Zahncreme, appretierte Binden etc. ${ }^{53}$. Hierzu gehörten auch zwei Arten «polar-biochemischer Nerven-Nahrung», deren eine «Lebenskraft gebend», also im Wortsinne «belebend», deren andere beruhigend wirken sollte ${ }^{54}$, der Brust-Honig-Sirup «Tussextra» und das Krebsmittel «Carcinomex».

47 «Durch die polar-biochemische Wechselwirkung der von der Erde zurückstrahlenden (reflektierten) elektromagnetischen Aufladungen und der [...] Spektralzonen des Sonnenlichtes entstehen entsprechend variierte, individuell von einander verschiedene Konstitutionsformen auch in der Organisation «Mensch». Dementsprechend unterscheiden wir unter der Zugrundelegung der im Zeugungs- und Geburtsquartal ineinander greifenden 〈Dreiklang-Wechselbeziehung〉 der bestimmten Spektralzonen des Sonnenlichtes vier verschiedene Konstitutionen: die Frühling-, Sommer-, Herbst- und Winter-Konstitution.» Schöpwinkel 1930, 50f.

48 Schöpwinkel 1930, 51 .

49 Schöpwinkel 1930, 52.

50 Schöpwinkel 1924, 176-178.

51 Schöpwinkel 1930, 52-58.

52 Der Mensch und sein Leben 1 1926, 43.

53 Schöpwinkel 1929b, 4f.

54 Schöpwinkel 1930, 80f. 
Acht Gesundheitstees und neun Massagecremes ergänzten das Sortiment weiter, für einzelne Indikationen wurden gefärbtes Licht ${ }^{55}$ und «Naturheilmaßnahmen» wie Bäder oder Umschläge empfohlen ${ }^{56}$.

Eine umfängliche «Verordnungs-Hinweis-Tabelle» gab zu einzelnen Indikationen Mittelempfehlungen aus den sechs Präparategruppen «Polar-biochemische Funktionsmittel», «Polar-biochemische Ergänzungspräparate» ${ }^{57}$, «Polar-biochemische Gesundheitstees», «Polar-biochemische Massagecremes», «Polar-biochemische Lichtfarben» und «Polar-biochemische Naturheilmittel», also physikalisch-therapeutische Massnahmen.

Schöpwinkel glaubte zudem, teilweise unter Berufung auf die Lehre Carl Huters (1861-1912), physiognomisch das Fehlen bestimmter Mineralien im Körper bestimmen zu können ${ }^{58}$, eine Vorstellung, die ebenfalls bis heute persistiert ${ }^{59}$.

Die in der Homöopathie übliche Dezimalpotenzierung lehnte er als artifiziell («polar-unbiologisch») ab und führte ein eigenes logarithmisches System ein, das er «polar-biochemisches Verdünnungsgesetz» nannte. Grundlagen hierfür entnahm er der Harmonielehre und Schwingungsgesetzen und schuf Reihen («Oktaven») aus jeweils acht Verdünnungsstufen im Verhältnis 1:2. Zur Begründung gab er an:

Auf Grund der in der Natur sich auswirkenden Gegensatzenergie (Bi-Polarität) ist die verhältniszahlenmäßige Stufenentfaltung (logarithmische Progression) durch das Paar bedingt, was bei Schall und Licht zu wechselbezüglich-achtstufigen Einklang-Organisationen (korrelativ-harmonischen Oktaven) führt. Das ist der Grund, weshalb jeder Künstler die Zahl 8 als mitfühlend angenehm (sympathisch), dagegen die Zahl 10 als mitfühlend unangenehm (unsympathisch) empfindet. ${ }^{60}$

\section{Mit dem Verfahren wollte er}

einen erkrankten Organismus mit dem Gleichmaß artgleicher Schwingungen von artgleichen Stoffen seinem ursprünglichen [...] Lebensgleichmaß wieder auf geradem Wege annähern ${ }^{61}$.

55 Etwa produziert durch Naturglasbirnen der «Samariter-Heillampen-Fabrik» in Karlsruhe, die in Schöpwinkels Schriften warb; vgl. Schöpwinkel 1929b, 112.

56 Schöpwinkel 1929b, 5-7. Für eine Zuordnung der Präparate und Empfehlungen zu einzelnen Indikationen siehe Schöpwinkel 1929b und Schöpwinkel 1931.

57 Nicht zu verwechseln mit den «Ergänzungsmitteln» im üblichen biochemischen Sprachgebrauch; diese waren bereits Bestandteil der ersten Gruppe.

58 So sollte «bei gleichzeitiger mürrisch-betrübter, sauer-griesgrämiger Miene und braun-gelbblassem, schrunzeligem, ausgezehrtem Gesicht» ein Mangel an Mangan, Lithium, Brom, Jod, Arsen, Natrium sulfuricum, Natrium phosphoricum und Silicea bestehen. Schöpwinkel 1929a, 134. Vgl. hierzu auch Schöpwinkel 1924, 65-109. Zu Huter und seinen heilkundlichen Vorstellungen vgl. Helmstädter 2004, 199-202.

59 Harnisch 1998, 41-47.

60 Schöpwinkel 1930, 64.

61 Schöpwinkel 1930, 64. 
Die einzelnen «polar-biochemischen Verdünnungsstufen» waren mit arabischen Ziffern (lg.P. 1-8) bezeichnet und wurden durch Angabe der jeweiligen «Oktave» in römischen Ziffern ergänzt. Die Angabe «lg.P. 8, II» bezeichnete also die achte Verdünnungsstufe der zweiten Oktave, was von der Konzentration her etwa mit D6 gleichzusetzen $w^{6}{ }^{62}$. Für die letztlich von ihm propagierten 20 Funktionsmittel gab es eine Kongruenztabelle zwischen der «früheren homoeopathischen Dezimal-Potenzierung» und der «jetzigen naturwissensch[aftlich] biologisch-biochem[isch]-logarithmisch-gesetzmässigen Potenzierung» ${ }^{63}$.

Bei der Komplexität des vorgeschlagenen Heilverfahrens verwundert es kaum, dass Schöpwinkel eine «Studiengemeinschaft für philosophisch-biologisch-biochemische Wissenschaften» begründete, deren Mitglieder sich durch ein dreijähriges «Studium» mit jährlichen Examina zum «geprüften Biochemiker» weiterbilden konnten. Zur Erlangung des Titels war eine schriftliche Abschlussarbeit erforderlich, zudem mussten durch amtlich beglaubigte Heilungsberichte von Patienten mindestens 50 «außergewöhnliche Heilungen» nachgewiesen werden ${ }^{64}$. In den «Studiengang» war auch der «Odopath» Peter Johannes Thiel (1864-1943) eingebunden, dessen Kurse in Anatomie, Physiologie, Pathologie und Augendiagnose für die Ausbildung zum «praktischen Biochemiker» angerechnet wurden. Thiel, der ein eigenes «odopathisches Heilsystem» aufgelegt hatte, fungierte ab 1.3.1926 sogar als «Verbands-Prüfungs-Kommissar» ${ }^{65}$.

\section{Weitere Nachfolger Schüßlers}

Demeter Georgiewitz Weitzer, genannt Surya (1873-1949), bekennender Anhänger okkulter Medizin und seinerseits Erfinder eines auf spagyrischen Prinzipien beruhenden «vereinfachten Heilsystems» ${ }^{66}$, betonte die «Beseelung» auch im anorganischen Bereich und formulierte im Zusammenhang mit «biochemischen» Salzen folgendes:

62 Ausführliche Tabelle bei Schöpwinkel 1930, 65 und 99, sowie Der Mensch und sein Leben 1 (1926) 213. Die Potenz lg.P. 8, III entsprach etwa C4; lg.P. 8, V etwa D15; lg.P. 8, VII etwa D21. 63 Schöpwinkel 1930, 99.

64 Zur Prüfungsordnung siehe Der Mensch und sein Leben 1 (1926), 30; a.a.O., 248-254 und 259-261, ist die Abschlussarbeit des «praktischen Biochemikers» Gerhard Lichtenberg aus Hirschberg/Schlesien abgedruckt. Er schrieb zum Thema «Die Dr. med. Schüßler'sche 〈biochemische Heilmethode〉 (Lebens-Chemie)».

65 Der Mensch und sein Leben 1 (1926) 81. Zur Prüfungskommission zählte auch der Spezialist für Hand- und Nageldiagnostik, Ernst Issberner-Haldane (1886-1966). Zu seinem Leben und Werk vgl. Miers 1970, 213. Zu Thiel vgl. Helmstädter 2004, 187-198.

66 Zu Suryas Heilsystem vgl. Helmstädter 2004, 202-205. 
Jedes Atom ist nicht nur ein elektromagnetisches Kraftfeld, nicht nur ein Planetensystem in nuce, nicht nur ein riesiges Energiereservoir, [...] sondern das Atom ist auch ein Zentrum vitaler oder biologischer Kräfte, ja im Grunde genommen nichts weiter als eine Offenbarung dieser lebendigen Kräfte oder eine Offenbarung des einen universellen Lebens. ${ }^{67}$

In diesem Sinne müsse der Begriff «Biochemie» erweitert werden und über eine rein funktionelle Substitutionstherapie analog der Pflanzendüngung hinausgehen ${ }^{68}$. Nachfolgend heisst es:

Ist das Blut krank oder fehlen ihm gewisse Substanzen, so kann die daraus entspringende Zelle nicht gesund und vollkräftig sein. [...] Den kranken, geschwächten Zellen neues Leben zuzuführen, ist also sicherlich ein Weg, der zur Gesundung führen kann, denn in den Körperzellen spielt sich das Leben ab. Doch man vergesse nie, die Körperzellen sind keineswegs das Leben selbst!

Der biochemische Arzt Gustav Grams, der unter anderem für die Anwendung biochemischer Komplexpräparate votierte, betonte zwar den Charakter der Schüßler-Salze als Substitutionstherapeutika, behandelte sie jedoch im Zusammenhang mit seinen Ausführungen zur dem Mesmerismus nahe stehenden «magnetischen Nervenmassage» ${ }^{69}$. Sein Namensvetter Konrad Grams hingegen beschränkte sich in Ausführungen zu seiner «KomplexBiochemie Kombi» ganz auf den substitutionstherapeutischen Aspekt, indem er den «Mineralsalzmangel unseres Blutes und der Gewebe» als «Grundursache der Krankheiten» begriff ${ }^{70}$.

Veröffentlichungen aus jüngerer Zeit stellen die Therapie wiederum ohne Zögern als biodynamistisch dar: «Sie [die Schüßler-Salze] senden uns lichtvolle, aktivierende, anfeuernde und schöpferische Lebensströme.» ${ }^{71}$ Ein Schüßler-Lehrbuch aus den 1990er Jahren trägt den Titel «Die Dr. SchüßlerMineraltherapie - Selbstheilung und Lebenskraft>. Dort wird postuliert, die Salze vermittelten «tiefgreifend in die körperlichen und psychischen Bereiche hineinwirkende Heilimpulse» und wirkten «auf der feinstofflichen Ebene selbst bei scheinbar ererbten Leiden und bei psychischem Krankheitsgeschehen $[\ldots] »^{72}$. Der Autor empfiehlt zur Auswahl des geeigneten Schüßler-Minerals auch metaphysische, auf hypothetischen Kraftemanationen beruhende Methoden wie den «kinesiologischen Muskeltest»oder

67 Surya $1923,159$.

68 Surya $1923,159$.

69 Grams 1922.

70 Grams 1928, 9. Grams, über den nichts Biographisches bekannt ist, hatte insgesamt 50 Biochemische Komplexmittel im Programm, die durch weitere 8 «Spezialpräparate Kombi» ergänzt wurden. Er gab eine «Volkstümliche Monatsschrift für Komplex-Biochemie und naturgemässe Heil- und Lebensweise» heraus.

71 Hausen 2003, 39.

72 Harnisch 1998, 24. 
das siderische Pendel ${ }^{73}$. Das biochemische Laboratorium BIKA vertrieb zu diesem Zweck eigens konstruierte technische Geräte. Der «biochemische Pendulator nach Dr. med. Carl E. Weiß» diente dazu, «vermittelst des Pendels mit wenigen Versuchen das im einzelnen Fall passende biochemische Mittel zu bestimmen» ${ }^{74}$. Ähnliches, nämlich «mittels der Pendeldiagnose das individuelle Konstitutionsmittel für jedermann» zu finden, sollte der «Zeregen-Apparat» leisten ${ }^{75}$.

\section{«Biochemie» als biodynamistisches Heilverfahren}

Die bereits bei Schüßler selbst, besonders allerdings bei seinen Nachfolgern zutage tretenden Vorstellungen einer therapeutischen Beeinflussung der «Lebenskraft» sind charakteristisch für eine grosse Gruppe komplementärmedizinischer Verfahren, die man als «biodynamistisch» bezeichnen kann. Ihnen ist eigen, Krankheit mit einem Nachlassen an «Lebenskraft» zu assoziieren und Therapien einzusetzen, die die «vis vitalis» kräftigen ${ }^{76}$. Sicherlich ist die Ansicht, alle komplementärmedizinischen Verfahren bedienten sich dieses Ansatzes ${ }^{77}$, nicht haltbar, doch gibt es eine grosse Anzahl «biodynamischer» Therapieverfahren ${ }^{78}$. In diesem Zusammenhang kamen und kommen auch immer wieder Arzneimittel zum Einsatz, deren Ziel es ist, Energie zu übertragen. So konnte gezeigt werden, dass die physikalischen Erscheinungen des Magnetismus, der Elektrizität, der Radioaktivität und des Lichtes ebenso zur Erklärung pharmakologischer Wirkungen herangezogen wurden wie hypothetische Kräfte, beispielsweise das «Od», ein vom Chemiker Karl Freiherr von Reichenbach (1788-1869) eingeführtes Energiepostulat ${ }^{79}$. Nicht zuletzt die Homöopathie ist dem Kreis der biodynamistischen Arzneithera-

73 Die Fähigkeit einer Person, die ein Schüßler-Präparat in der Hand hält, Druck auf den ausgestreckten Arm durch Muskelspannung auszugleichen, wird als Mass für die Eignung des Präparates hergenommen. Pendel ziehen angeblich über geeigneten Schüßler-Salzen grössere Kreise. Vgl. hierzu Harnisch 1998, 48f. Ähnliches wurde auch für Zimpel-Spagyrika behauptet, deren Strahlungsemanation ebenfalls mit dem siderischen Pendel nachgewiesen worden sein soll: Weiss 1930.

74 BIKA 1930, 28.

75 Das Gerät war in zwei Ausführungen, «in schön poliertem Holzkasten» oder lediglich «in solidem Pappkasten mit Leinwandbezug», erhältlich. BIKA 1930, 29.

76 Der vielschichtige, historisch schwer zu fassende, aber gleichwohl unmittelbar verständliche Begriff der «Lebenskraft» soll an dieser Stelle nicht diskutiert werden. Vgl. hierzu beispielsweise die Übersichten von Gottlieb 1943; ders. 1953; Lohff 1981; Botsch 1997 und Brown 1999.

77 So ausdrücklich Turnheim 2002.

78 Jütte 1996, 16.

79 Helmstädter 2004. 
pien zuzuordnen. Hahnemann benutzte den Begriff der «Lebenskraft` gleichwohl beiläufig wie selbstverständlich ${ }^{80}$.

Die hohen Marktanteile der Homöopathie und verwandter Verfahren sowie die Tatsache, dass nach wie vor eine Vielzahl «energetischer» Heilverfahren neu beworben werden ${ }^{81}$, zeigen, dass nach wie vor die Bereitschaft der Patienten besteht, sich auf Therapieansätze einzulassen, denen eine immaterielle Pathophysiologie zugrunde liegt. Dass auch die vordergründig unter dem Aspekt einer Substitutionstherapie mit organischen Salzen eingeführte Schüßlersche «Biochemie» zur Zeit unter der Vorstellung, die mineralischen Zubereitungen stärkten die «Lebenskraft ${ }^{82}$, eine Art Renaissance erlebt, muss auf den ersten Blick erstaunen. Das Verfahren war jedoch nie eine reine Substitutionstherapie, denn Schüßler betonte bereits in frühen Auflagen seiner «abgekürzten Therapie» die Notwendigkeit kleiner Gaben ${ }^{83}$ und bekannte sich stets zur Homöopathie. Seine Nachfolger betonten dann zunehmend immaterielle Aspekte der Wirkungsvermutung. Das Verfahren reiht sich somit nahtlos in eine Vielzahl komplementärmedizinischer Verfahren ein, die mit einem biodynamistischen Ansatz werben, und dürfte seine Beliebtheit vor allem diesem Aspekt, weniger seinen physiologisch-chemischen Grundlagen auf dem Stand des 19. Jahrhunderts, verdanken.

\section{Bibliographie}

Ashmore, Malcolm, "The theatre of the blind: starring a promethean prankster, a phony phenomenon, a prism, a pocket, and a piece of wood", Social Studies of Science 23 (1993) 67-106

BIKA Laboratorium (Hrsg.), Verzeichnis der Erzeugnisse des biochemischen Laboratoriums «IKA»-Zentrale für Biochemie (Stuttgart o.J. [ca. 1930])

Biochemische Central-Officin, Der biochemische Wegweiser (Heft 1) (Bremen o.J. [ca. 1920])

Bischof, Marco, Biophotonen. Das Licht in unseren Zellen (Frankfurt ${ }^{9} 1998$ )

Borchardt, Albert, «Die 〈biochemische Heilkunst〉 des Dr. Wilhelm Schüssler», in: Jürgen Reichling/Wolf-Dieter Müller-Jahncke/Albert Borchardt, Arzneimittel der komplementären Medizin (Eschborn 2001) 63-70

Botsch, Walter, Die Bedeutung des Begriffs Lebenskraft für die Chemie zwischen 1750 und 1850 (Diss. phil. Stuttgart 1997)

Brown, Guy, The energy of life (London 1999)

80 Eine Stichwortsuche in der jetzt vorliegenden elektronischen Gesamtausgabe seiner Werke liefert 328 über das ganze Werk versteckte Fundstellen, das gedruckte Gesamtregister von Minder 2002 immerhin über 100. Bereits Gottlieb 1943, 23f., und ders. 1953 bezweifelt trotz mancher theoretischer Ungereimtheiten die grundsätzliche Akzeptanz des Lebenskraftphänomens in der Homöopathie nicht. Zur Ansicht Hahnemanns vgl. § 9-11 des «Organon $:$ Hahnemann ed. Haehl 1979, 44f.

81 Vgl. Helmstädter 2004, 299-304.

82 Vgl. Harnisch 1998, Helmke Hausen 2003, Feichtinger/Niedan-Feichtinger 2004.

83 Schüßler 1875,6 . 
Deters, Hermann, Handbuch der Dr. Schüsslerschen Biochemie. Die Dr. Schüsslersche Biochemie, eine wissenschaftliche Ionen-, Reiz-, Konstitutions- und Nährsalzlehre (Radeburg 1926)

Feichtinger, Thomas/Susana Niedan-Feichtinger, Schüßler für Körper und Seele (Heidelberg 2004)

Gäbler, Hartwig, Wesen und Anwendung der Biochemie. Therapie mit Mineralstoffen nach Dr. Schüßler (Karlsruhe 1988)

Gefken, Gisela, Dr. med. Wilhelm Heinrich Schüßler. Ein Literaturverzeichnis (Oldenburg 1998)

Gottlieb, B[ernward] J[osef], Das Problem des Lebendigen im ärztlichen Weltbild: G.E. Stahl, Hahnemann und Virchow (Leipzig 1943)

Gottlieb, Josef, «Die Lehre von der Lebenskraft bei Hahnemann», Sudhoffs Archiv 37 (1953) $150-252$

Grams, Gustav, Die Heilkraft des Magnetismus und der magnetischen Nervenmassage bei allen nervösen Leiden durch Übertragung der Lebenskraft unter Berücksichtigung der biochemischen Heilmittel (Regensburg 1922)

Grams, Konrad, Handbuch der Komplex-Biochemie (Berlin ${ }^{3} 1928$ )

Habacher, Maria, «Auf der Suche nach dem 〈Od〉. Karl Ludwig Freiherr von Reichenbach (17881869) und Karl Wilhelm Mayrhofer (1806-1853), zwei Verbündete contra Justus von Liebig», Clio Medica 14 (1980) 105-118

Hahnemann, Samuel, Organon der Heilkunst, hg. von Richard Haehl (1921, Nachdruck Stuttgart 1979)

Harnisch, Günter, Die Dr. Schüßler-Mineraltherapie. Selbstheilung und Lebenskraft (Bietigheim 31998)

Helmke Hausen, Monika, Lebensquell Schüßlersalze (München 2003)

Helmstädter, Axel, Medizin für die Lebenskraft. Arzneilicher Biodynamismus als Konzept der medikamentösen Komplementärmedizin (Habil.-Schrift Marburg 2004)

Jütte, Robert, Geschichte der Alternativen Medizin. Von der Volksmedizin zu den unkonventionellen Therapien von heute (München 1996)

Karrasch, Bertram, Volksheilkundliche Laienverbände im Dritten Reich (Stuttgart 1998)

Lampert, [Heinrich], Umstrittene Heilverfahren. Anwendungsmöglichkeiten in Klinik und Praxis (Stuttgart 1953)

Lohff, Brigitte, «Zur Geschichte der Lehre von der Lebenskraft», Clio Medica 16 (1981) 101-112

Miers, Horst E., Lexikon des Geheimwissens (Freiburg 1970)

Minder, Peter, Gesamtregister zu Hahnemanns Werk. Sach-, Arznei- und Personenverzeichnis (Stuttgart 2002)

Platz, Hugo, Dr. Schüßler und seine biochemische Heilmethode. Ein Gedenkbuch zu seinem 100. Geburtstag (Leipzig 1921)

Reiff, [Albert], Gesammelte Beweise aus der Literatur für die Richtigkeit der Mineralsalztherapie, 6. Tsd. (Oldenburg 1924)

- Gesammelte Beweise aus der Literatur für die Richtigkeit der Mineralsalztherapie, 9.-14. Tsd. (Oldenburg 1932)

Schling-Brodersen, Uschi, «Biochemie», in: Werner E. Gerabek et al. (Hrsg.), Enzyklopädie Medizingeschichte (Berlin 2005) 182f.

Schöpwinkel, D[ietrich], Die Biochemie als Weltgesetz. Eine solar-biochemische KonstitutionsLehre und ihre wissenschaftliche Begründung. Biochemische Welterkenntnis, Bd. 1 (Mülheim 1924)

- Die Polar-Biochemie als Weltgesetz. Eine polar-biochemische Konstitutions-Lehre und ihre wissenschaftliche Begründung. Polar-Biochemische Welterkenntnis, Bd. 1 (Düsseldorf/Mülheim ${ }^{2} 1929$ a)

- Die Dr. med. Schüßler-Schöpwinkel'sche Polar-Biochemie. Ein kurzer Leitfaden zur Einführung in die Grundgedanken der praktischen Auswertung der in: "Die Polar-Biochemie als Weltgesetz» niedergelegten biologisch-polar-biochemischen Gesetzmäßigkeit. Polar-biochemische Welterkenntnis, Bd. 2. (Mülheim ${ }^{5} 1930$ )

- Fach-Vorlesungen über die Forschungs-Fortschritte der polar-biochemischen Natur-Heilwissenschaft. 3. Fachvorlesung (Bad Honnef 1937)

Schöpwinkel, Dieter, Verordnungs-Hinweis-Tabelle der polar-biochemischen Heil-Wissenschaft. Polar-biochemische Welterkenntnis, Bd. 3 (Düsseldorf/Mülheim 1929b) 
[Schöpwinkel, Dietrich], Kleiner Ratgeber der Polar-Biochemie (Düsseldorf o.J. [ca. 1931])

Schüßler, Wilhelm Heinrich, «Eine abgekürzte homöopathische Therapie», in: Allgemeine Homöopathische Zeitung 86 (1873) 91f.

Schüßler, [Wilhelm Heinrich], Eine abgekürzte Therapie, gegründet auf Histologie und CellularPathologie (Oldenburg ${ }^{2} 1875$ )

- Eine abgekürzte Therapie. Biochemische Behandlung der Krankheiten (Oldenburg ${ }^{9} 1882$ )

- Eine abgekürzte Therapie. Anleitung zur biochemischen Behandlung der Krankheiten (Oldenburg/Leipzig ${ }^{37} 1911$ )

Surya, G. W. (i.e. Weitzer, Demeter Georgiewitz), Homöopathie, Isopathie, Biochemie, Iatrochemie und Elektrohomöopathie. Okkulte Medizin, VIII (Berlin 1923)

Teich, Mikulas, «Die Suche der Biochemie nach Identität», in: Erika Hickel (Hrsg.), Biochemische Forschung im 19. Jahrhundert-mit einer Bibliographie der Quellen (Braunschweig 1989) (Braunschweiger Veröffentlichungen zur Geschichte der Pharmazie und der Naturwissenschaften, 32)

Turnheim, Klaus, «Alternative und naturwissenschaftliche Therapieformen: unvereinbare Gegensätze», Wiener Medizinische Wochenschrift 114 (2002) 958-962

Ulpts, Jürgen, Die Geschichte der Naturheilweise Biochemie (Oldenburg 1998)

Vöckel, Anja, Die Anfänge der physiologischen Chemie: Ernst Felix Immanuel Hoppe-Seyler (1825-1895) (Diss. phil. Berlin 2003)

Weiss, Karl Erhard, «Zur Pharmakologie der spagyrisch-homöopathischen Arzneimittel nach Zimpel», Zeitschrift für Spagyrik 1 (1930) 139-149

Winckelmann, Joachim, ABC der Geheimwissenschaften (Berlin 1956)

Winter, York, Die Biochemie des Oldenburger Arztes Wilhelm Heinrich Schüßler (1821-1898) (Diss. med. Göttingen 1971)

Zankl, Heinrich, Fälscher, Schwindler, Scharlatane. Betrug in Forschung und Wissenschaft (Weinheim 2003) 